\title{
Antimicrobial Sensitivity Testing of Bacterial Isolates from Subclinical Mastitis in Buffaloes
}

\author{
Rakesh Kumar $^{1^{*}}$, Yudhbir Singh Rana ${ }^{2}$ and Anshu Sharma ${ }^{3}$ \\ ${ }^{1}$ Department of Animal Husbandry \& Dairying, Haryana, India \\ ${ }^{2}$ Department of Veterinary Medicine, LUVAS, Hisar, Haryana, India \\ ${ }^{3}$ Incharge College Central Laboratory, COVSc, LUVAS, Hisar, Haryana, India \\ *Corresponding author
}

\section{A B S T R A C T}

\section{Keywords \\ Antibiotics, \\ Antimicrobial sensitivity, \\ Buffalo, Mastitis \\ Article Info \\ Accepted: \\ 06 September 2018 \\ Available Online: \\ 10 October 2018}

In the present investigation, in-vitro antimicrobial sensitivity of 46 isolates from milk of mastitis affected buffaloes towards 21 antimicrobials revealed variation in their sensitivity pattern. Overall all strains of staphylococci and streptococci were sensitive to penicillin G, chloramphenicol, cloxacillin, ampicillin, kanamycin, enrofloxacin, neomycin, oleandomycin, ampiclox, spiramycin, novabiocin and ciprofloxacin antibiotics.

\section{Introduction}

Mastitis is a one of the most economically important disease of dairy animals, which not only causes heavy economic losses in terms of milk quality and quantity but also poses public health hazard due to persistence of antibiotic residues in the milk. Due to the wide and indiscriminate use of antimicrobial agents for treatment of Mastitis, there are chances of emergence of resistant bacterial strains and change in sensitivity pattern of microbes; Therefore, it is necessary to perform repeated drug trials with newer formulations. The selection of antimicrobial for a particular pathogen should be based on culture and sensitivity testing.

\section{Materials and Methods}

A total of 235 quarter milk samples collected from 59 apparently healthy lactating Murrah buffaloes from Buffalo Research Centre, CCS Haryana Agricultural University, Hisar were the source of material for the study.

Different strains of various organisms isolated from udder infections were subjected to invitro chemotherapeutic sensitivity using 21 antimicrobials by disc diffusion method as described by Bauer et al., (1966). A small amount of growth from isolated colonies of organisms with the help of platinum loop was transferred in to tubes of trypticase soya broth and incubated for two to five hours at $37^{\circ} \mathrm{C}$ so 
as to obtain a turbidity, equivalent to that obtained by adding $0.5 \mathrm{ml}$ of $0.048 \mathrm{M} \mathrm{Bacl}_{2}$ $\left(1.17 \% \mathrm{BaCl}_{2} .2 \mathrm{H}_{2} \mathrm{O}\right)$ to $99.5 \mathrm{ml}$ of 0.36 $\mathrm{NH}_{2} \mathrm{SO}_{4}(1 \% \mathrm{v} / \mathrm{v})$. The broth culture with the help of sterile cotton swab was then evenly spread by smearing over the surface of blood agar and Nutrient agar plates.

The discs were then placed on the agar and pressed gently with a sterile forceps so as to have a uniform close contact with the medium.

The plates were then kept at low temperature for three to five minutes to allow pre-diffusion of antibiotics. The plates were then incubated at $37^{\circ} \mathrm{C}$ for $18-24 \mathrm{hrs}$ and observed for sensitivity by measuring the zone of inhibition. Results were recorded on the basis of table provided by the manufacturer for zone size interpretation as sensitive (S) and resistant (R).

\section{Results and Discussion}

Results of in-vitro antimicrobial sensitivity of all bacterial isolates from SCM cases of mastitis against 21 antimicrobial agents are presented in the table below:

All the 35 strains of staphylococci were sensitive to penicillin G, chloramphenicol, cloxacillin, ampicillin, kanamycin, enrofloxacin, neomycin, oleandomycin, ampiclox, spiramycin, novobiocin and ciprofloxacin. More than 80 per cent of these strains showed sensitivity towards oxytetracycline, tetracycline, erythromycin, amoxycillin, amoxyclav, co-trimoxazole and streptomycin, however sensitivity to nitrofurantoin and polymyxin-B was 62.85 and 74.28 per cent, respectively.

All the 9 strains of diphtheroids were found sensitive to oxytetracycline, tetracycline, chloramphenicol, cloxacillin, enrofloxacin, neomycin, oleandomycin, amoxyclav, ampiclox, and novobiocin. More than 80 percent of these strains were found sensitive to penicillin G, ampicillin, kanamycin, amoxycillin, spiramycin, ciprofloxacin and streptomycin. Sensitivity towards nitrofurantoin, polymyxin-B, erythromycin and co-trimoxazole ranged between 44.44 and 77.77 per cent.

Both the strains of streptococci showed sensitivity towards nitrofurantoin, penicillin G, oxytetracycline, tetracycline, chloramphenicol, cloxacillin, ampicillin, kanamycin, enrofloxacin, neomycin, oleandomycin, erythromycin, amoxycillin, amoxyclav, co-trimoxazole, spiramycin, novobiocin, ciprofloxacin and streptomycin. Sensitivity towards polymyxin-B was found to be 50 per cent of these strains.

The studies of in-vitro antimicrobials sensitivity testing of different bacterial isolates from SCM cases of mastitis against 21 antimicrobial agents showed that all the strains of staphylococci were sensitive to penicillin G, chloramphenicol, cloxacillin, ampicillin, kanamycin, enrofloxacin, neomycin, oleandomycin, ampiclox, spiramycin, novobiocin and ciprofloxacin. More than 80 per cent of these strains showed sensitivity towards oxytetracycline, tetracycline, erythromycin, amoxycillin, amoxyclav, cotrimoxazole and streptomycin, however sensitivity to nitrofurantoin and polymyxin-B was 62.85 and 74.28 per cent, respectively. Similar results for chloramphenicol have been reported by Dahiya and Kapur (1984), Saxena et al., (1993), Mallikarjunaswami and Krishnamurthy (1997), Saxena (2000) and Bulla et al., (2003). RamaniPushpa et al., (1998), Gentilini et al., (2000), Datta and Ranganekar (2001) and Kumari et al., (2002) suggested highest sensitivity of these isolates to gentamycin. Saxena et al., (1993) found penicillin-G least sensitive against udder isolates. 
Antimicrobial sensitivity of microorganisms (46) isolated from subclinical cases of mastitis

\begin{tabular}{|c|c|c|c|c|c|c|c|}
\hline \multirow{4}{*}{$\begin{array}{l}\text { Antimicrobial } \\
\text { agent } \\
\text { Nitrofurantoin } \\
\text { (NF) } \\
(300 \mathrm{mcg})\end{array}$} & \multicolumn{7}{|c|}{ Organism and number tested } \\
\hline & \multicolumn{3}{|c|}{$\begin{array}{c}\text { Staphylococci } \\
(35)\end{array}$} & \multicolumn{2}{|c|}{$\begin{array}{c}\text { Streptococci } \\
\text { (2) }\end{array}$} & \multicolumn{2}{|c|}{$\begin{array}{c}\text { Diphtheroids } \\
(9)\end{array}$} \\
\hline & $\mathrm{S}$ & 22 & $(62.85)$ & 2 & $(100.00)$ & 6 & $(66.66)$ \\
\hline & $\mathrm{R}$ & 13 & $(37.15)$ & 0 & $(00.00)$ & 3 & $(33.33)$ \\
\hline $\begin{array}{l}\text { Penicillin-G } \\
\text { (P) }\end{array}$ & $\mathrm{S}$ & 35 & $(100.00)$ & 2 & $(100.00)$ & 8 & $(88.88)$ \\
\hline 10 units & $\mathrm{R}$ & 0 & $(0.00)$ & 0 & $(00.00)$ & 1 & $(11.11)$ \\
\hline $\begin{array}{l}\text { Oxytetracycline } \\
\text { (O) }\end{array}$ & $\mathrm{S}$ & 34 & $(97.14)$ & 2 & $(100.00)$ & 9 & $(100.00)$ \\
\hline (30 mcg) & $\mathrm{R}$ & 1 & $(2.85)$ & 0 & $(00.00)$ & 0 & $(0.00)$ \\
\hline $\begin{array}{l}\text { Tetracycline } \\
\text { (T) }\end{array}$ & $\mathrm{S}$ & 33 & $(94.28)$ & 2 & $(100.00)$ & 9 & $(100.00)$ \\
\hline (30 mcg) & $\mathrm{R}$ & 2 & $(5.71)$ & 0 & $(00.00)$ & 0 & $(0.00)$ \\
\hline $\begin{array}{l}\text { Chloramphenicol } \\
\text { (C) }\end{array}$ & $\mathrm{S}$ & 35 & (100.00) & 2 & $(100.00)$ & 9 & (100.00) \\
\hline$(30 \mathrm{mcg})$ & $\mathrm{R}$ & 0 & $(0.00)$ & 0 & $(0.00)$ & 0 & $(0.00)$ \\
\hline $\begin{array}{l}\text { Cloxacillin } \\
(\mathrm{Cx})\end{array}$ & $\mathrm{S}$ & 35 & (100.0) & 2 & $(100.00)$ & 9 & (100.00) \\
\hline (10 mcg) & $\mathrm{R}$ & 0 & $(0.00)$ & 0 & $(0.00)$ & 0 & $(0.00)$ \\
\hline $\begin{array}{l}\text { Ampicillin } \\
\text { (A) }\end{array}$ & $\mathrm{S}$ & 35 & (100.00) & 2 & $(100.00)$ & 8 & $(88.88)$ \\
\hline (10 mcg) & $\mathrm{R}$ & 0 & $(0.00)$ & 0 & $(0.00)$ & 1 & (11.11) \\
\hline $\begin{array}{l}\text { Kanamycin } \\
(\mathrm{K})\end{array}$ & $\mathrm{S}$ & 35 & $(100.00)$ & 2 & $(100.00)$ & 8 & (88.88) \\
\hline (30 mcg) & $\mathrm{R}$ & 0 & $(0.00)$ & 0 & $(0.00)$ & 1 & (11.11) \\
\hline $\begin{array}{l}\text { Polymyxin B } \\
(\mathrm{Pb})\end{array}$ & $\mathrm{S}$ & 26 & (74.28) & 1 & $(50.00)$ & 4 & $(44.44)$ \\
\hline (300 mcg) & $\mathrm{R}$ & 9 & $(25.71)$ & 1 & $(50.00)$ & 5 & $(55.55)$ \\
\hline $\begin{array}{l}\text { Enrofloxacin } \\
(\mathrm{Ex})\end{array}$ & $\mathrm{S}$ & 35 & (100.00) & 2 & $(100.00)$ & 9 & (100.00) \\
\hline$(10 \mathrm{mcg})$ & $\mathrm{R}$ & 0 & $(0.00)$ & 0 & $(0.00)$ & 0 & $(0.00)$ \\
\hline
\end{tabular}




\begin{tabular}{|c|c|c|c|c|c|c|}
\hline $\begin{array}{l}\text { Neomycin } \\
\text { (N) }\end{array}$ & $S$ & $35 \quad(97.22)$ & 2 & (100.00) & 9 & (100.00) \\
\hline (30 mcg) & $\mathrm{R}$ & $(0.00)$ & 0 & (00.00) & 0 & $(0.00)$ \\
\hline $\begin{array}{l}\text { Oleandomycin } \\
(\mathrm{Ol})\end{array}$ & $S$ & $35(100.00)$ & 2 & (100.00) & 9 & (100.00) \\
\hline$(15 \mathrm{mcg})$ & $\mathrm{R}$ & $(0.00)$ & 0 & $(0.00)$ & 0 & $(0.00)$ \\
\hline $\begin{array}{l}\text { Erythromycin } \\
\text { (E) }\end{array}$ & $\mathrm{S}$ & $32 \quad(91.42)$ & 2 & (100.00) & 7 & (77.77) \\
\hline$(15 \mathrm{mcg})$ & $\mathrm{R}$ & (8.57) & 0 & $(0.00)$ & 2 & (22.22) \\
\hline $\begin{array}{l}\text { Amoxycillin } \\
(\mathrm{Am})\end{array}$ & $\mathrm{S}$ & $34 \quad(97.14)$ & 2 & (100.00) & 8 & $(88.88)$ \\
\hline (30 mcg) & $\mathrm{R}$ & $(2.85)$ & 0 & $(0.00)$ & 1 & (11.11) \\
\hline $\begin{array}{l}\text { Amoxyclav } \\
\text { (Ac) }\end{array}$ & $\mathrm{S}$ & $34 \quad$ (97.14) & 2 & (100.00) & 9 & (100.00) \\
\hline (30 mcg) & $\mathrm{R}$ & $(2.85)$ & 0 & $(0.00)$ & 0 & $(0.00)$ \\
\hline $\begin{array}{l}\text { Ampiclox } \\
(\mathrm{Ax})\end{array}$ & $\mathrm{S}$ & 35 (100.00) & 2 & (100.00) & 9 & (100.00) \\
\hline (30 mcg) & $\mathrm{R}$ & $(0.00)$ & 0 & $(0.00)$ & 0 & $(0.00)$ \\
\hline $\begin{array}{l}\text { Co-trimoxazole } \\
\text { (Co) }\end{array}$ & $\mathrm{S}$ & $31 \quad(88.57)$ & 2 & (100.00) & 7 & (77.77) \\
\hline$(25 \mathrm{mcg})$ & $\mathrm{R}$ & $4 \quad(11.42)$ & 0 & $(0.00)$ & 2 & $(22.22)$ \\
\hline $\begin{array}{l}\text { Spiramycin } \\
\text { (Sr) }\end{array}$ & $\mathrm{S}$ & $35(100.00)$ & 2 & (100.00) & 8 & (88.88) \\
\hline (30 mcg) & $\mathrm{R}$ & $(0.00)$ & 0 & $(0.00)$ & 1 & (11.11) \\
\hline $\begin{array}{l}\text { Novobiocin } \\
(\mathrm{Nv})\end{array}$ & $\mathrm{S}$ & 35 (100.00) & 2 & (100.00) & 9 & (100.00) \\
\hline$(30 \mathrm{mcg})$ & $\mathrm{R}$ & $(0.00)$ & 0 & $(0.00)$ & 0 & $(0.00)$ \\
\hline $\begin{array}{l}\text { Ciprofloxacin } \\
\text { (Cf) }\end{array}$ & $\mathrm{S}$ & $35(100.00)$ & 2 & (100.00) & 8 & (88.88) \\
\hline$(10 \mathrm{mcg})$ & $\mathrm{R}$ & $(0.00)$ & 0 & $(0.00)$ & 1 & (11.11) \\
\hline $\begin{array}{l}\text { Streptomycin } \\
\text { (S) }\end{array}$ & $\mathrm{S}$ & $34 \quad(97.14)$ & 2 & (100.00) & 8 & (88.88) \\
\hline$(10 \mathrm{mcg})$ & $\mathrm{R}$ & $(2.85)$ & 0 & $(0.00)$ & 1 & (11.11) \\
\hline
\end{tabular}

S-Sensitive, R=Resistant 
However higher sensitivity against various antibiotics have been reported by Dahiya and Kapur (1984), Tuteja (1999) and Bulla et al., (2003) whereas Kalorey et al., (1983), Bhalerao et al., (2000) and Saxena (2000) reported low efficacy of various antimicrobials to staphylococci.

All the strains of diphtheroids were found sensitive to oxytetracycline, tetracycline, chloramphenicol, cloxacillin, enrofloxacin, neomycin, oleandomycin, amoxyclav, ampiclox, and novobiocin. Both the strains of streptococci showed sensitivity towards nitrofurantoin, penicillin $\mathrm{G}$, oxytetracycline, tetracycline, chloramphenicol, cloxacillin, ampicillin, kanamycin, enrofloxacin, neomycin, oleandomycin, erythromycin, amoxycillin, amoxyclav, co-trimoxazole, spiramycin, novobiocin, ciprofloxacin and streptomycin. Sensitivity of other microbials were in the range of 44.44 to 77.77 per cent. Almost similar findings were recorded by Verma and Mishra (1977). Lower sensitivity of ampilox (56.25\%) was observed by Saxena (2000), Bertoldini et al., (1985) and Tuteja (1999) also reported the similar sensitivity to spiramycin and co-trimoxazole.

These results indicate the variability in the antimicrobial sensitivity pattern for microbes isolated from SCM cases and suggest the importance of epidemiologic knowledge of a dairy herd to guide initial therapy.

In-vitro antimicrobial sensitivity of 46 bacterial isolates from SCM cases of mastitis was determined against 21 antimicrobial agents. All the strains of staphylococci were sensitive to penicillin $\mathrm{G}$, chloramphenicol, cloxacillin, ampicillin, kanamycin, enrofloxacin, neomycin, oleandomycin, ampiclox, spiramycin, novobiocin and ciprofloxacin. More than 80 per cent of these strains showed sensitivity towards oxytetracycline, tetracycline, erythromycin, amoxycillin, amoxyclav, co-trimoxazole and streptomycin, however sensitivity to nitrofurantoin and polymyxin-B was 62.85 and 74.28 per cent, respectively.

All the strains of diphtheroids were found sensitive to oxytetracycline, tetracycline, chloramphenicol, cloxacillin, enrofloxacin, neomycin, oleandomycin, amoxyclav, ampiclox, and novobiocin. More than 80 percent of these strains were found sensitive to penicillin G, ampicillin, kanamycin, amoxycillin, spiramycin, ciprofloxacin and streptomycin. Sensitivity towards nitrofurantoin, polymyxin-B, erythromycin and co-trimoxazole ranged between 44.44 and 77.77 per cent.

Both the strains of streptococci showed sensitivity towards nitrofurantoin, penicillin $\mathrm{G}$ oxytetracycline, tetracycline, chloramphenicol, cloxacillin, ampicillin, kanamycin, enrofloxacin, neomycin, oleandomycin, erythromycin, amoxycillin, amoxyclav, co-trimoxazole, spiramycin, novobiocin, ciprofloxacin and streptomycin. Sensitivity towards polymyxin-B was found to be 50 per cent of these strains.

\section{References}

Bauer, A.W., Kirby, W.M.M., Sherris, J.C. and Turck, M. 1966. Antibiotic susceptibility testing by a standardized single disc method. Am. J. Clin. Path. 45: 493.

Bertoldini, G., Brunner, F. and Redaelli, G. 1985. Further studies on the antibiotic sensitivity of streptococci and staphylococci associated with bovine mastitis. Archivio Vet Italiano., 36: 94101 (Abstr. Vet. Bull. 56: 1432).

Bhalerao, D.P., Jagadish, S., Keskar, D.V., Dangore, A.D. and Sharma, L.K. 2000. Antibiogram and treatment of bovine 
subclinical mastitis. Indian Vet., J.77: 244-246.

Bulla, T.R., Rana, Y.S. and Sharma, A. 2003. Antimicrobial sensitivity of bacteria isolated from subclinical mastitis in buffaloes. Presented at $4^{\text {th }}$ Asian Buffalo Congress on "Buffalo for security and rural employment" held at New Delhi, INDIA from Feb. 25-28, 2003.

Dahiya, B.S. and Kapur, M.P. 1984. Bacteriological examination and therapeutic trials with the cloxacillin sodium in clinical cases of mastitis. Indian J. Vet. Med. 4: 1-4.

Dutta, S. and Rangnekar, A. 2001. Subclinical mastitis in a jersey herd. Indian vet. $J$. 78: 161-162.

Gentilini, E., Denamiel, G., Liorente, P., Godaly, S., rebuelto, M. and DeGregoriot. 2000. Antimicrobial sensitivity of Staphylococcus aureus isolated from bovine mastitis in Argentina. J. Dairy Sc.83: 1224-1227.

Kalorey, D.R., Purohit, J.H. and Dholakia, P.M. 1983. Studies on the incidence of sub-clinical mastitis, its etiology and in vitro sensitivity of isolates. Indian $\mathrm{J}$. Anim. Sci. 53: 961-963.

Kumari, P; Gupta, M. and JanakiRam, B. 2002. Diagnosis and therapy of subclinical mastitis in post-parturient cows. Indian vet. J. 79: 79.

Millikarjunaswami, M.C. and Krishnamurthy, G.V. 1997. Antibiogram of bacterial pathogen isolated from bovine subclinical mastitis cases. Indian Vet. $J$. 74(10): 885-886.

RamaniPushpa, R.N., Venketa Reddy, K. and Ramaline Raju, S. 1998. Antibiogram of bacteria isolated from mastitis cases of cattle in Nallore District. Indian Vet. J.75: 352-353.

Saxena, R.K., Dutta, G.N., Barah, P. and Buragohain, Jyoti 1993. Drug susceptibility and treatment of bovine subclinical mastitis. Indian Vet. J. 70: 201-203.

Saxena, Vivek 2000. Studies on prevalence, detection and control of subclinical mastitis in buffaloes. M.V.Sc. Thesis CCS HAU, Hisar, pp. 111.

Tuteja, F.C. 1999. Studies on mastitis in buffaloes with special reference to serum selenium status and control by treating teat canal infections. Ph.D. Thesis, Haryana Agric. Univ., Hisar.

Verma, S.P. and Misra, S.S. 1977. In vitro sensitivity of isolated organisms from bovine mastitis to different antimicrobials. Indian vet. J. 54: 834839.

\section{How to cite this article:}

Rakesh Kumar, Yudhbir Singh Rana and Anshu Sharma. 2018. Antimicrobial Sensitivity Testing of Bacterial Isolates from Subclinical Mastitis in Buffaloes. Int.J.Curr.Microbiol.App.Sci. 7(10): 529-534. doi: https://doi.org/10.20546/ijcmas.2018.710.058 